\title{
High resolution diffusion-weighted imaging with readout segmentation of long variable echo-trains for determining myometrial invasion in endometrial carcinoma
}

Mengnv Xie ${ }^{1 \dagger}$, Zhen Ren ${ }^{1 \dagger}$, Dujun Bian², Dan Li ${ }^{2}, \mathrm{Li} \mathrm{Y}^{1}$, Fang Zhu' ${ }^{1}$, Rui Huang ${ }^{1}$, Zhibang Zhang ${ }^{1}$, Suye Suye and Chun $\mathrm{Fu}^{1 *}$

\begin{abstract}
Background: We assessed the image quality of endometrial cancer lesions by readout segmentation of long variable echo-trains (RESOLVE) diffusion-weighted imaging (DWI) compared with that by single-shot echo-planar imaging (SS-EPI) DWI, aimed to explore the value of RESOLVE DWI for determining myometrial invasion and clinical stage in endometrial cancer.

Materials and methods: From April 2017 to March 2018, a total of 30 endometrial cancer patients (mean age $52.8 \pm 9.0$ years), who had undergone RESOLVE DWI and SS-EPI DWI, were included in the study. The image quality of endometrial carcinoma by two kinds of DWI scanning methods was compared qualitatively and quantitatively. The Spearman rank correlation test was used to assess the correlation of qualitative image quality scores between two readers. The accuracy of two DWI methods in detecting myometrial invasion and staging of endometrial carcinoma was calculated according to postoperative pathological results. The indexes were analyzed including sensitivity, specificity, accuracy, positive predictive value (PPV), and negative predictive value (NPV).

Results: The qualitative score of RESOLVE DWI group was superior to SS-EPI DWI group in every aspect of five aspects (all $P<0.001$ ). Interobserver agreement of depiction was good or excellent in two DWI sequences. Signal to noise ratio and contrast to noise ratio values in RESOLVE DWI group were both higher than those in SS-EPI DWI group $(P<0.001)$. No statistical difference of apparent diffusion coefficient value was observed between two DWI groups $(P=0.261)$. The specificity, accuracy, PPV, and NPV of estimating myometrial invasion by RESOLVE DWI in three cases (intramucosal lesion, $<50 \%$ superficial invasion and $\geq 50 \%$ deep invasion) were all higher than those by SS-EPI DWI for endometrial carcinoma. Especially RESOLVE DWI was valuable in judging $<50 \%$ superficial invasion (95\%Cl:0.586, 0.970). No significant difference in accuracy staging was between the two DWI groups $(P=0.125)$.

Conclusion: RESOLVE DWI can provide higher quality images of endometrial carcinoma than SS-EPI DWI. The highquality images are helpful for precise assessment of myometrial invasion in endometrial cancer.
\end{abstract}

Keywords: Magnetic resonance imaging, RESOLVE DWI, Myometrial invasion, Endometrial carcinoma

\footnotetext{
* Correspondence: fuchun0814@csu.edu.cn

${ }^{\dagger}$ Mengnv Xie and Zhen Ren contributed equally to this work.

'Department of Obstetrics and Gynecology, The Second Xiangya Hospital,

Central South University, Changsha, No.139 Renmin Road, Changsha, Hunan

410011, PR China

Full list of author information is available at the end of the article
}

\section{$\triangle B M C$}

(c) The Author(s). 2020 Open Access This article is licensed under a Creative Commons Attribution 4.0 International License, which permits use, sharing, adaptation, distribution and reproduction in any medium or format, as long as you give appropriate credit to the original author(s) and the source, provide a link to the Creative Commons licence, and indicate if changes were made. The images or other third party material in this article are included in the article's Creative Commons licence, unless indicated otherwise in a credit line to the material. If material is not included in the article's Creative Commons licence and your intended use is not permitted by statutory regulation or exceeds the permitted use, you will need to obtain permission directly from the copyright holder. To view a copy of this licence, visit http://creativecommons.org/licenses/by/4.0/ The Creative Commons Public Domain Dedication waiver (http://creativecommons.org/publicdomain/zero/1.0/) applies to the data made available in this article, unless otherwise stated in a credit line to the data. 


\section{Background}

Endometrial cancer is one of the most common malignant tumor threatening to female health [1]. The incidence of endometrial cancer is second-ranked in female genital tract tumors only lower to cervical cancer in China. Preoperative evaluation of endometrial cancer is very important for tailoring treatment. The purpose of preoperative evaluation is mainly to evaluate intrauterine lesions, with emphasis on the depth of myometrial invasion and the extent of local spread [2]. Several imaging methods have been used to evaluate intrauterine lesions for endometrial cancer. Which method is more suitable for the evaluation of endometrial cancer is still in the exploration.

Magnetic resonance imaging (MRI) has been widely accepted for the evaluation of myometrial invasion and extrauterine tumor spread owing to its superior contrast resolution and excellent soft tissue differentiation [3]. However, conventional MRI sequences like T1-weighted imaging (T1WI) and T2-weighted imaging (T2WI) which lacks specific parameters regarding tumor microstructure or biological information have limitations in assessing myometrial invasion. Functional MRI techniques like diffusion-weighted MRI (DW-MRI) and dynamic contrast-enhanced MRI (DCE-MRI) can improve the accuracy of myometrial invasion. Diffusion-weighted imaging (DWI) is commonly used in pelvic scanning. Multiple studies of endometrial cancer have shown that DWI images and apparent diffusion coefficient (ADC) values are helpful to evaluate the depth of myometrial invasion [4-8]. The accuracy and sensitivity of assessing myometrial invasion by DWI are higher than those by DCE-MRI in endometrial cancer [9].

DWI has been used as an adjunct to conventional MRI in the evaluation of endometrial cancer lesions by providing quantitative information about water molecular diffusion, but its disadvantage is leading degradation of image quality [10]. Therefore, the improvement of the scanning sequence has been the key to DWI image quality. Single-shot echo-planar imaging (SS-EPI) is a commonly used scanning sequence of DWI, but artifacts and blurring effects may occur due to the long time of data acquisition and accumulated phase errors [11]. Readoutsegmented echo-planar imaging (RS-EPI) is a new DWI scan sequence, which can shorten the sampling time and reduce the accumulation of phase errors in the direction of phase encoding so that a high-resolution DWI images can be generated [12]. DWI using RS-EPI is also called readout segmentation of long variable echo-trains diffusion-weight imaging (RESOLVE DWI) (Response comment 1). RESOLVE DWI has been used in the brain, spine, breast, and kidney imaging [13-16]. Its application in pelvic is mainly concentrated in rectum, prostate and bladder imaging [17-19].
Therefore, this study aimed to apply RESOLVE DWI to endometrial cancer. The study had two purposes. The primary was to compare the image quality of endometrial cancer lesions with RESOLVE DWI and SS-EPI DWI. The secondary was to evaluate the value of RESOLVE DWI in determining myometrial invasion and clinical stage of endometrial cancer.

\section{Materials and methods \\ Patients}

The comparative study of two scanning methods was approved by the Institutional Review Board. Written informed consent was obtained from all patients before treatment. 34 patients with pre-operative pathological diagnoses of endometrial cancer were collected between April 2017 and March 2018. Four patients were excluded: (1) contraindications of MRI examination with claustrophobia and metal objects in the body such as metal stents, cardiac pacemakers, and intrauterine devices $(n=2)$; (2) receiving antineoplastic therapy before operation after pelvic MRI examination $(n=2) .30$ patients (mean age, 52.8 \pm 9.0 years; range, $32-75$ years) were finally included in our study (Fig. 1). Each patient received T1WI, T2WI, contrast-enhanced T1WI, RESOLVE DWI, and SS-EPI DWI.

\section{MRI protocol}

All MRI examinations were performed at $3.0 \mathrm{~T}$ MRI scanner (Magnetom Skyra, Siemens, Erlangen, Germany). The coil specifications were standard 16channel phased-array body coil and 32-channel phasedarray spinal coil. The scan ranges from the upper margin of the ilium to the symphysis pubis. All patients received MRI examinations in the non-menstrual period. Each patient had a fasting for $6 \mathrm{~h}$ before examination and kept the bladder adequately filled.

Scan sequences contained turbo spin-echo (TSE) T1WI, TSE T2WI, contrast-enhanced TSE T1WI, RESOLVE DWI, and SS-EPI DWI. TSE T1WI was acquired in axial planes. Fat suppressed TSE T2WI were acquired in three orthogonal planes (axial, sagittal, and coronal). Immediately after bolus injection of Gadopentetate Dimeglumine (Gd-DTPA) with a dose of $0.1 \mathrm{mmol} / \mathrm{kg}$ (Magnevist, Schering, Berlin, Germany), fat-suppressed TSE T1WI were obtained in the axial, sagittal, and coronal planes. All patients underwent SS-EPI and RS-EPI DWI scanning of pelvic cross-section, respectively. Meanwhile, images reconstruction of diffusion sensitive gradient $\mathrm{b}$ value $0 \mathrm{~s} / \mathrm{mm}^{2}$ and $1000 \mathrm{~s} / \mathrm{mm}^{2}$ was completed. The protocol parameters were shown in Table 1.

\section{Image analysis}

All the measurements and evaluations were conducted by Siemens 3.0 T Skyra MR work station (version syngo 


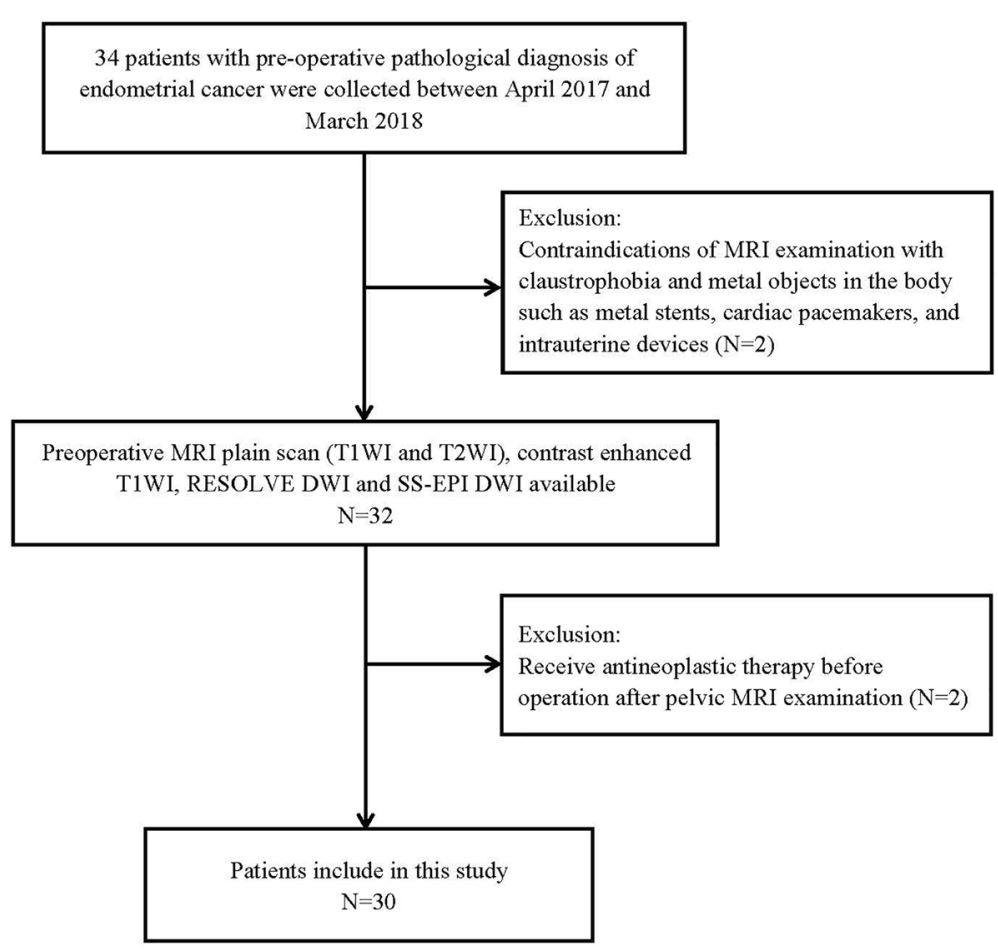

Fig. 1 Illustration of the flow diagram of included patients

MR D13). The MRI images were reviewed independently by two senior radiologists respectively with 10 years and 12 years of working experience and were blinded to the histopathology reports. Any discrepancies were resolved by consensus. The analytic content was as follows: qualitative and quantitative assessments of image quality, the depth of myometrial invasion, and the preoperative stage.

\section{Qualitative evaluation of image quality}

Based on the related literature, the study firstly analyzed the quality of MRI images from four aspects (b $1000 \mathrm{~s} / \mathrm{mm}^{2}$ ). These four aspects were geometric distortion, image blurring, ghosting artifacts, and lesion conspicuity [20]. The score degree of each aspect was divided into a 6-point scale (ranging from 1 to 6, listed in Table 2) [21]. The scores of the aforementioned four items were further divided into a

Table 1 Imaging parameters for T1-,T2- and contrast-enhanced T1-weighted MRI and SS-EPI, and RESOLVE DWI sequences

\begin{tabular}{|c|c|c|c|c|c|c|c|c|c|}
\hline \multirow{2}{*}{$\begin{array}{l}\text { Parameters } \\
\text { Scan plane }\end{array}$} & \multirow{2}{*}{$\begin{array}{l}\text { T1WI } \\
\text { axial }\end{array}$} & \multicolumn{3}{|c|}{ Fat-suppressed T2WI } & \multicolumn{3}{|c|}{ Contrast-enhanced T1WI } & \multirow{2}{*}{$\begin{array}{l}\text { SS-EPI DWI } \\
\text { axial }\end{array}$} & \multirow{2}{*}{$\begin{array}{l}\text { Resolve DWI } \\
\text { (RS-EPI) DWI } \\
\text { axial }\end{array}$} \\
\hline & & axial & sagittal & coronal & axial & sagittal & coronal & & \\
\hline Slice thickness, mm & 4 & 4 & 4 & 4 & 4.5 & 4 & 4.5 & 4 & 4 \\
\hline Slice gap, mm & 0.4 & 0.4 & 0.4 & 0.4 & 0.45 & 0.4 & 0.45 & 0.4 & 0.4 \\
\hline $\mathrm{TR}, \mathrm{ms}$ & 500 & 5460 & 4450 & 5460 & 507 & 582 & 500 & 6800 & 5700 \\
\hline $\mathrm{TE}, \mathrm{ms}$ & 12 & 87 & 87 & 87 & 12 & 9 & 9 & 113 & 78 \\
\hline Matrix & $320 \times 320$ & $320 \times 320$ & $320 \times 320$ & $320 \times 320$ & $320 \times 320$ & $320 \times 320$ & $320 \times 320$ & $192 \times 192$ & $200 \times 200$ \\
\hline $\mathrm{FOV}, \mathrm{mm}$ & $300 \times 300$ & $300 \times 300$ & $280 \times 280$ & $280 \times 280$ & $240 \times 240$ & $240 \times 240$ & $260 \times 260$ & $300 \times 300$ & $300 \times 300$ \\
\hline Flip angle & $160^{\circ}$ & $148^{\circ}$ & $156^{\circ}$ & $148^{\circ}$ & $160^{\circ}$ & $180^{\circ}$ & $180^{\circ}$ & - & - \\
\hline $\mathrm{b}$ value, $\mathrm{s} / \mathrm{mm}^{2}$ & - & - & - & - & - & - & - & $0 ; 1000$ & $0 ; 1000$ \\
\hline Average & 2 & 2 & 2 & 2 & 2 & 2 & 2 & 2 & 2 \\
\hline Scan times & $2 \min 14 s$ & $2 \mathrm{~min} 37 \mathrm{~s}$ & $2 \min 44 s$ & $2 \min 14 s$ & $2 \min 52 s$ & $3 \min 32 s$ & $3 \min 14 s$ & $2 \min 9 s$ & $4 \min 59 s$ \\
\hline
\end{tabular}

MRI magnetic resonance imaging, SS-EPI single-shot echo-planar imaging, RESOLVE readout segmentation of long variable echo-trains, DWI diffusion-weighted imaging, T1WI T1-weighted imaging, T2WI T2-weighted imaging, RS-EPI readout-segmented echo-planar imaging, TR time of repeat, TE time of echo, FOV field of vision, min minutes, $s$ second 
Table 2 Detailed rules for qualitative analysis of image quality

\begin{tabular}{|c|c|c|c|c|c|c|}
\hline \multirow[t]{2}{*}{ Parameter } & \multicolumn{6}{|l|}{ Score } \\
\hline & 6 & 5 & 4 & 3 & 2 & 1 \\
\hline $\begin{array}{l}\text { Geometric } \\
\text { distortion }\end{array}$ & $\begin{array}{l}\text { no } \\
\text { distortion }\end{array}$ & $\begin{array}{l}\text { probably no } \\
\text { distortion }\end{array}$ & $\begin{array}{l}\text { faint severe } \\
\text { distortion }\end{array}$ & $\begin{array}{l}\text { partially severe } \\
\text { distortion }\end{array}$ & $\begin{array}{l}\text { severe } \\
\text { distortion }\end{array}$ & $\begin{array}{l}\text { extremely severe } \\
\text { distortion }\end{array}$ \\
\hline Image blurring & no blurring & probably no blurring & faint severe blurring & partially severe blurring & severe blurring & extremely severe blurring \\
\hline Ghosting artifacts & no artifact & probably no artifact & faint severe artifact & partially severe artifact & severe artifact & extremely severe artifact \\
\hline Lesion conspicuity & outstanding & good & above average & below average & poor & unacceptable \\
\hline $\begin{array}{l}\text { Overall image } \\
\text { quality }\end{array}$ & outstanding & good & above average & below average & poor & unacceptable \\
\hline
\end{tabular}

3 -point scale as follows: for items with a score of 5-6, accumulate 2 points; $3-4$ points, accumulate 1 point; $1-2$ points, accumulate 0 point. Finally, the radiologist accumulated the scores of four items to get the overall image quality scores. A score of 8 is the best and 0 is the worst.

\section{Quantitative evaluation of image quality}

The quantitative analysis included the measurement of signal to noise ratio (SNR), contrast to noise ratio (CNR) and ADC. The measurement method on RESOLVE DWI is shown in Fig. $2\left(b=1000 \mathrm{~s} / \mathrm{mm}^{2}\right)$. Each index was measured three times for each case, and the average was taken as its final value. SNR was defined by the following formula: SNR = SI (lesion)/SD (background) [22]. SI (lesion) represented the signal intensity (SI) of the lesion. SD (background) referred to the standard deviation of the SI of background noise. The region of interest (ROI) with the minimum size of $10 \mathrm{~mm}^{2}$ was obtained on the axial sections. All ROIs were put on the same slice on which the maximum area of the endometrial carcinoma shown. The selected ROIs of SI (lesion) were consistent with that of ADC value measurement in the same patient. CNR was defined by the following relationships: $\mathrm{CNR}=(\mathrm{SI}$ (lesion) $-\mathrm{SI}$ (myometrium)) $/ \mathrm{SD}$ (background) [23]. SI (lesion) and SI (myometrium) represented the SI of the lesion and myometrium, respectively. The ROI of myometrium was selected as the same size and layer of the lesion. ADC was defined by the following formula: $\mathrm{ADC}=\mathrm{In}\left(\mathrm{SI}_{0} / \mathrm{SI}_{1}\right) /\left(\mathrm{b}_{1}-\mathrm{b}_{0}\right) . \mathrm{b}_{1}=1000 \mathrm{~s} /$ $\mathrm{mm}^{2}, \mathrm{~b}_{0}=0 \mathrm{~s} / \mathrm{mm}^{2} . \mathrm{SI}_{1}$ and $\mathrm{SI}_{0}$ represented the SI of the lesion at $\mathrm{a} b$ value of $1000 \mathrm{~s} / \mathrm{mm}^{2}$ and $0 \mathrm{~s} / \mathrm{mm}^{2}$, respectively [15]. Freehand ROIs of RESOLVE DWI were carefully matched to the SS-EPI in the same patient.

\section{MRI appearance of myometrial invasion and preoperative staging of endometrial cancer}

T2WI and DWI were evaluated together in order to determine the depth of the myometrial invasion and preoperative stage of endometrial cancer. Simultaneous assessment of the findings of T2WI and DW images was defined as "T2-DWI". Two radiologists both of whom were blinded to the histopathological reports assessed the T2-RESOLVE DWI and T2-SS-EPI DWI images with 2 weeks apart between two sequences and evaluated the depth of myometrial invasion. Differences in the assessment were resolved by means of consensus. The method of judging the degree of myometrial invasion in the study was described by Nougaret S [24]. A line was drawn along the expected inner edge of the myometrium (endometrium-myometrium junction) on axial oblique images acquired perpendicular to the endometrium. Then, 2 lines are drawn: one measuring the thickness of the entire myometrium an another measuring the maximum tumor extent within the myometrium. The ratio of the 2 lines detailed represents the percentage of myometrial invasion. The depth of myometrial invasion were divided into 2 categories according to the FIGO staging system (2009) (Supplementary Table 1). The presence of myometrial invasion limited to the endometrial cavity or less than $50 \%$ was considered to be as IA and the presence of equal to or more than $50 \%$ myometrial invasion was considered to be as IB. For more detailed analysis, myometrial infiltration was divided into three cases as followers: intramucosal lesion, less than 50\% superficial invasion and equal to or more than $50 \%$ myometrial invasion (Supplementary Table 2).

\section{Reference standard}

All patients underwent total hysterectomy, bilateral salpingo-oophorectomy, and pelvic nodal dissection with or without para-aortic nodal dissection. A specialized gynecology/oncology pathologist with 20 years of experience performed the histopathologic examination of all samples. The final histologic result was available for each patient and constituted the reference standard for comparison. Depth of myometrial invasion (superficial invasion, confined to endometrium or inner half of the myometrium; deep invasion, invading the outer half of the myometrium), presence of cervical stromal invasion, and presence of metastasis within the sampled lymph nodes were confirmed microscopically. The staging standard of endometrial cancer patients was the International Federation of Obstetrics and Gynecology (FIGO) 2009 criteria. 

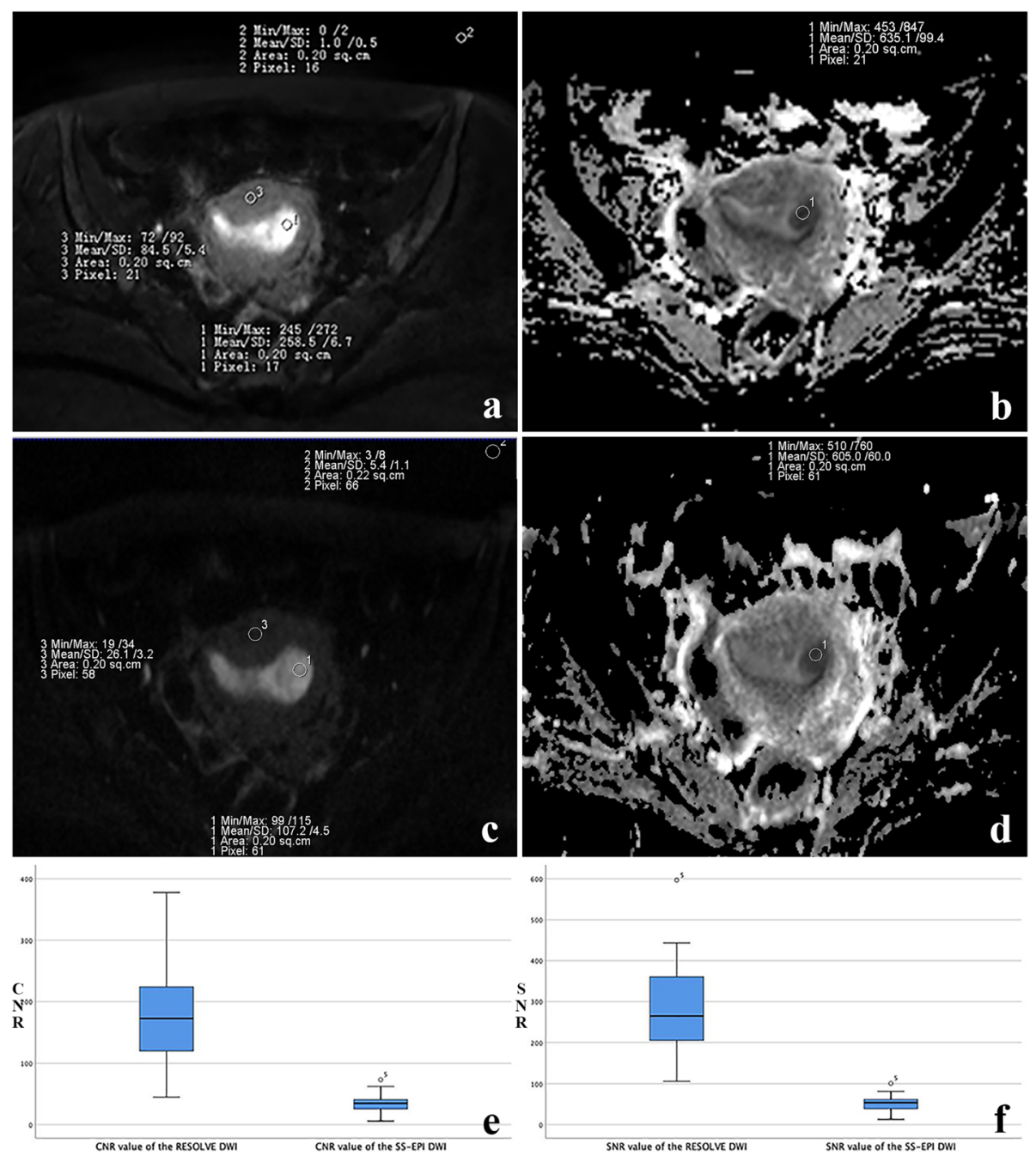

Fig. 2 Acquisition value of signal to noise ratio (SNR), contrast to noise ratio (CNR) and apparent diffusion coefficient (ADC) on cross-sectional MRI $\left(b=1000 \mathrm{~s} / \mathrm{mm}^{2}\right)$. a SNR and CNR measurement on readout segmentation of long variable echo-trains diffusion-weight imaging (RESOLVE DWI) image. ROI 1, the signal intensity (SI) of the lesion (SI lesion); ROI 2, SI of background noise (SI background); ROI 3, SI of the myosphere of the uterus (SI myosphere). b ADC value measurement of lesion on ADC maps of RESOLVE DWI. ROI 1, the ADC value of the lesion. The mean SNR, CNR and ADC values are 517, 348 and $635.1 \times 10^{-6} \mathrm{~mm}^{2} / \mathrm{s}$, respectively. c SNR and CNR measurement on single-shot echo-planar imaging diffusion-weighted imaging (SS-EPI DWI) image. ROI 1, the signal intensity (SI) of the lesion (SI lesion); ROI 2, SI of background noise (SI background); ROI 3, SI of the myosphere of the uterus (SI myosphere). d ADC value measurement of lesion on ADC maps of SS-EPI DWI. ROI 1, the ADC value of the lesion. The mean SNR, CNR and ADC values are $97.5,73.7$ and $605 \times 10^{-6} \mathrm{~mm}^{2} / \mathrm{s}$, respectively. e Box plot shows significant differences were found in CNR values between the RESOLVE DWI and DWI group $(P<0.05)$. $\mathbf{f}$ The SNR values of the RESOLVE DWI group was significantly higher than that of the DWI group $(P<0.05)$

\section{Statistical analysis}

All statistics were performed using SPSS 25.0. Differences among the data were considered statistically significant at $P<0.05$. The SNR, CNR, ADC value and qualitative scores of image quality were compared between the RESOLVE DWI and SS-EPI DWI group respectively. The Paired t-test was used for continuous variables (SNR and CNR). The Wilcoxon's signed-rank method for non-normal Distribution Variables (ADC and qualitative scores of image quality).
The Spearman rank correlation test was used to assess the correlation of qualitative image quality scores between two readers. The correlation coefficient rho $\left(r_{a}\right)$ was obtained to compare the degree of correlation as follows: little or no relationship if $0 \leq \mathrm{r}_{\mathrm{a}}<0.2$, fair if $0.2 \leq$ $\mathrm{r}_{\mathrm{a}}<0.4$, moderate if $0.4 \leq \mathrm{r}_{\mathrm{a}}<0.6$, good if $0.6 \leq \mathrm{r}_{\mathrm{a}}<0.8$, and excellent if $0.8 \leq \mathrm{r}_{\mathrm{a}}$. The accuracy of myometrial invasion and preoperative staging of endometrial cancer by RESOLVE DWI and SS-EPI DWI was calculated according to pathological results. The difference between the RESOLVE DWI and SS-EPI DWI group was tested 


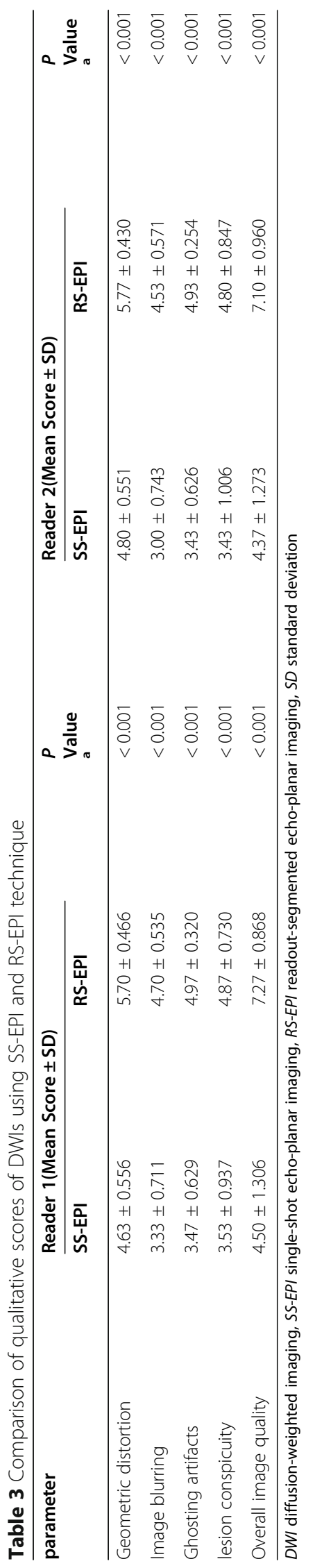


by Fisher's exact probability method. The sensitivity, specificity, accuracy, positive predictive value (PPV), and negative predictive value (NPV) of judgment myometrial invasion by RESOLVE DWI and SS-EPI DWI were also calculated based on pathological results.

\section{Results}

\section{Clinical features}

Of the 30 patients in the study, 16 were postmenopausal with median menopause time 5.5 years (range, 1-20 years). All patients underwent operation within 2 weeks after the MRI examination. The types of operations were summarized in (Supplementary Table 3). The cases of FIGO stage IA, IB, II, IIIA, IIIB and IV were $21,4,1,1$, 2,1 , respectively. Five pathological types were described as follows: endometrial adenocarcinoma (23 cases), adenosquamous carcinoma (3 cases), endometrial mucinous adenocarcinoma (1 case), serous papillary adenocarcinoma (1 case) and carcinosarcoma ( 2 cases).

\section{Qualitative analysis of image quality}

According to the scan sequence, the obtained data were divided into two groups: RESOLVE DWI and SS-EPI DWI. The qualitative scores of two evaluators in Table 3 showed each aspect in the RESOLVE DWI group was better than that in the SS-EPI DWI group (all $P<0.001$ ).
Interobserver agreement of the depiction of geometric distortion, image blurring, ghosting artifacts, lesion conspicuity and overall image quality were good or excellent in two DWI sequences (RESOLVE: 0.671, 0.674, 0.83, 0.796, 0.835; SS-EPI: 0.634, 0.644, 0.947, 0.873, 0.668, Supplementary Table 4). Examples of the advantages of RESOLVE DWI were presented in Figs. 3 and 4.

\section{Quantitative analysis of image quality}

The average values of SNR for SS-EPI DWI and RESOLVE DWI were $51.37 \pm 18.37$ and $279.46 \pm 109.48$, respectively. The SNR values of the RESOLVE DWI were higher than those of SS-EPI DWI $(P<0.001,95 \%$ CI: 191.28-264.89, Fig. 2). The average values of CNR were significantly higher for RESOLVE DWI $(176.12 \pm 72.72)$ than those for SS-EPI DWI $(34.72 \pm 15.36)(P<0.001$, 95\% CI: 117.39-165.41, Fig. 2). The average ADC values for SS-EPI DWI and RESOLVE DWI were $(0.920 \pm$ $0.055) \times 10^{-3} \mathrm{~mm}^{2} / \mathrm{s}$ and $(0.890 \pm 0.052) \times 10^{-3} \mathrm{~mm}^{2} / \mathrm{s}$, respectively. No statistical difference in ADC value was observed between SS-EPI DWI and RESOLVE DWI $(P=0.261)$.

\section{Myometrial invasion of endometrial carcinoma}

Diagnostic test evaluation indexes for judging myometrial invasion were summarized in Table 4. Only the
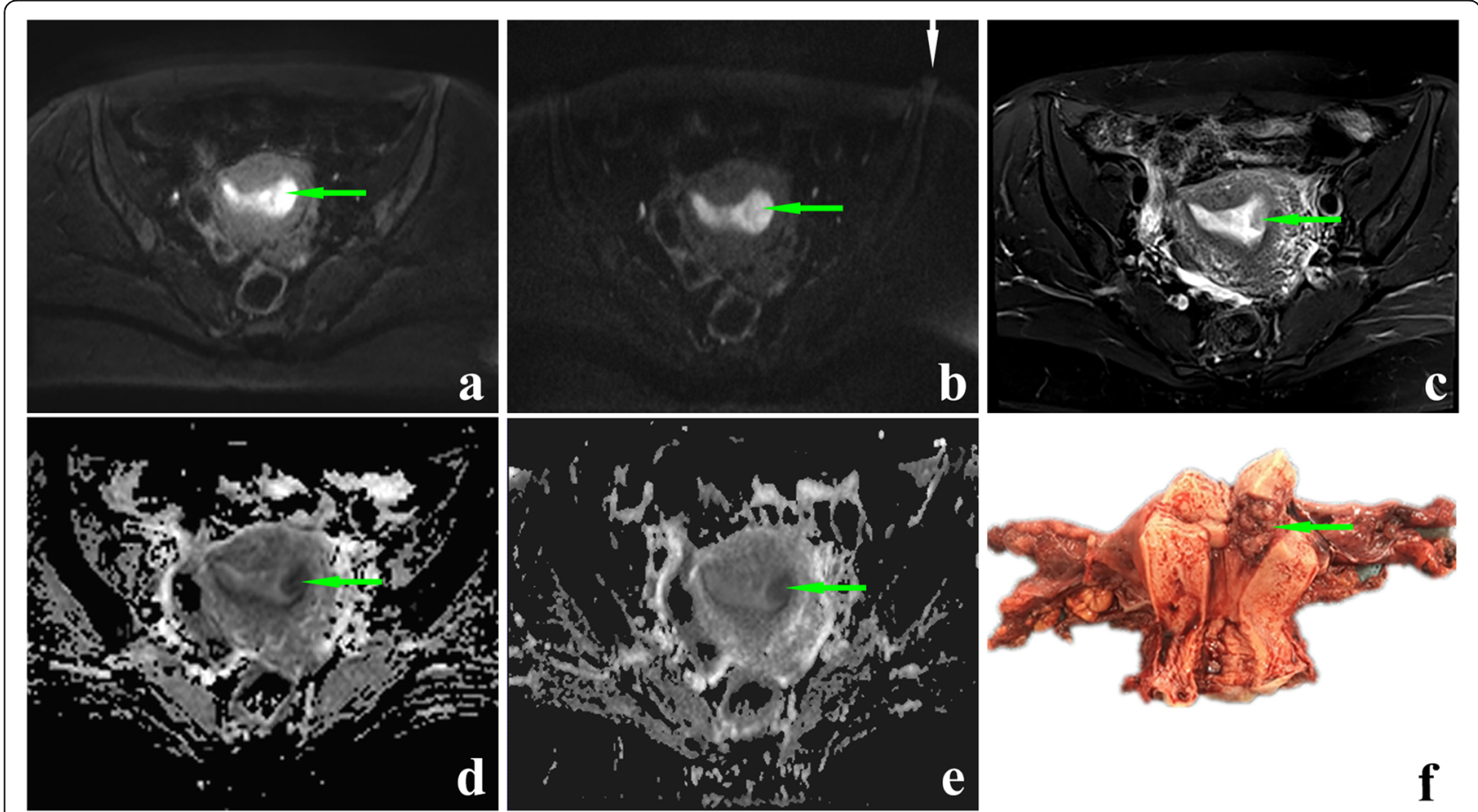

Fig. 3 A 46-year-old patient with IA stage endometrial cancer. The endometrial cancer lesion on axial RESOLVE DWI image (a, green arrow), SSEPI DWI image (b, green arrow), T2-weighted image (c, green arrow), ADC map corresponding to RESOLVE DWI sequence (d, green arrow) and ADC map corresponding to SS-EPI DWI sequence (e, green arrow). The b value of all the images is $1000 \mathrm{~s} / \mathrm{mm}^{2}$. The lesion can be seen more clearly on (a) than on (b). Geometric distortion can be seen on (b) (white arrow). $\mathbf{f}$ The postoperative view of the specimen. The invasion depth of endometrial carcinoma lesion was less than half of the thickness of myometrium 

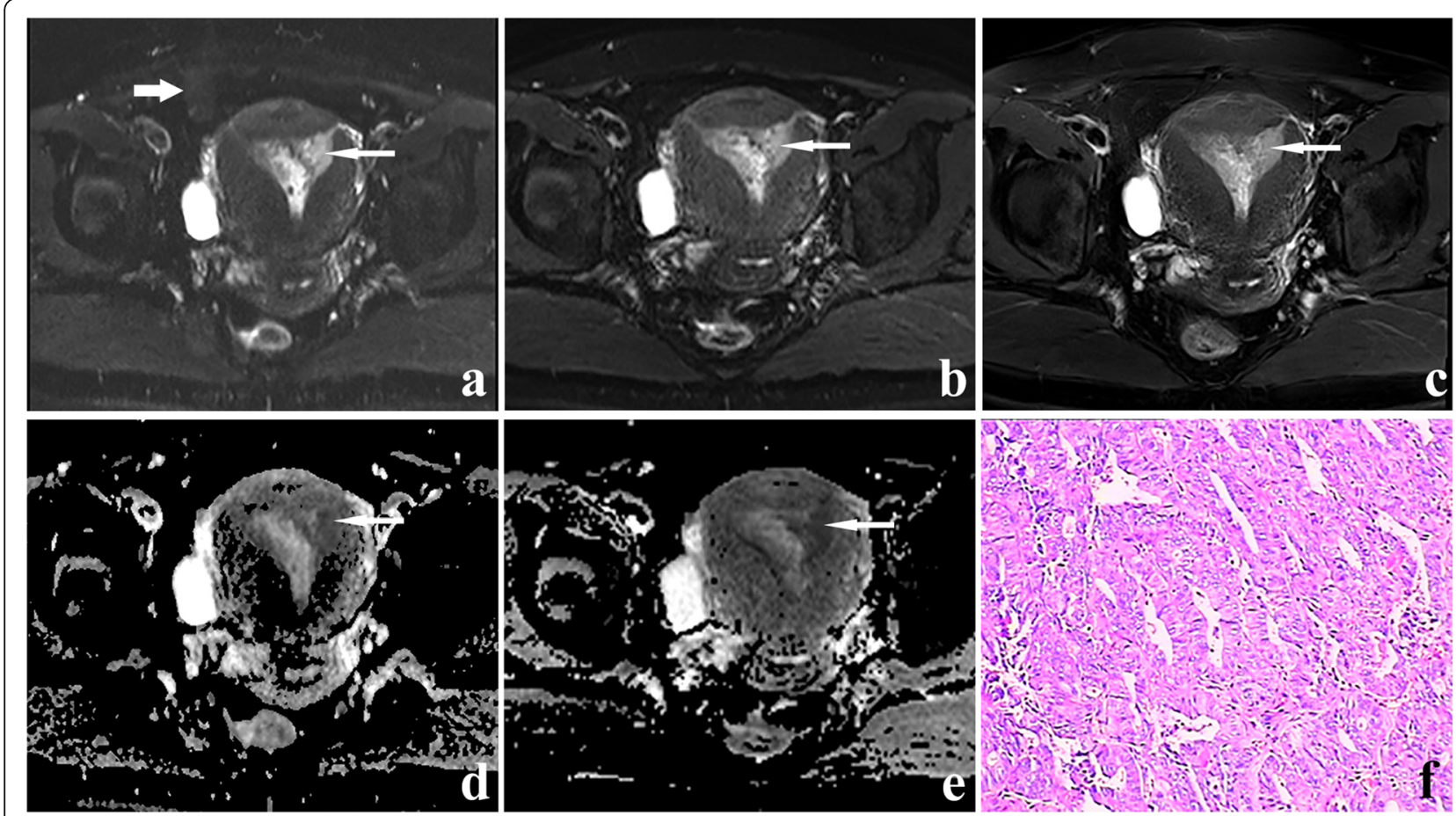

Fig. 4 A 47-year-old patient with stage IA endometrial carcinoma. The lesion (thin white arrow) on axial SS-EPI DWI image (a), RESOLVE DWI image (b), T2-weighted image (c), ADC map corresponding to SS-EPI DWI (d) and ADC map corresponding to RESOLVE DWI (e). The b value of all the images is $1000 \mathrm{~s} / \mathrm{mm}^{2}$. Artifacts were distinct on (a) (thick arrow). The hyperintense endometrial cancer lesion can be seen more clearly on (b) than on (a). $\mathbf{f} H \&$ E staining ( $\times 100$ magnification). The pathological diagnosis was well-differentiated endometrioid adenocarcinoma

sensitivities of assessing intramucosal lesion by the two scanning methods were the same. The sensitivity of assessing myometrial invasion in the other two cases by RESOLVE DWI were both higher than those by SS-EPI DWI. The specificity, accuracy, PPV and NPV of estimating myometrial invasion in three cases by RESOLVE DWI were all higher than those by SS-EPI DWI in endometrial carcinoma. We further analyzed 95\% confidence interval of sensitivity and PPV. We found that RESOLVE DWI was valuable in judging $<50 \%$ superficial invasion. The positive likelihood ratios of RESOLVE DWI group in three cases were all higher than those of the SS-EPI DWI group. The negative likelihood ratios of the RESOLVE DWI group in three cases were all lower than those of the SS-EPI DWI group.

\section{Stage of endometrial carcinoma}

The accuracy of preoperative staging by RESOLVE DWI and SS-EPI DWI were $86.67 \%$ (26/30) and 66.67\% (20/ 30 ) in endometrial cancer, respectively (Table 5 ). The results showed no significant difference in accuracy staging was between the two methods $(P=0.125) .4$ cases were wrongly staged by RESOLVE DWI and 10 cases were by SS-EPI DWI. The details were as follows: IA misdiagnosed as IB (RESOLVE: 1 case; SS-EPI: 3 cases), IB misdiagnosed as IA (RESOLVE: 1; SS-EPI: 3), II misdiagnosed as IA (SS-EPI:1), IIIA misdiagnosed as IB (RESOLVE: 1; SS-EPI: 1), and IIIB case misdiagnosed as II (RESOLVE: 1; SS-EPI: 2) (Supplementary Table 5). One case of stage IIIB endometrioid adenocarcinoma was accurately diagnosed by RESOLVE DWI (shown in Fig. 5).

\section{Discussion}

Accurate preoperative evaluation of endometrial cancer lesions is very important for conservative treatment [9]. This also helps choose the appropriate approach of surgery and monitoring treatment effect. Our study confirmed that RESOLVE DWI technology can provide high-quality MR images of endometrial cancer lesions. The high-quality images are helpful for precise assessment of myometrial invasion of endometrial cancer.

The increased cellularity of endometrial cancer causes restricted diffusion, which leads to increased signal on the DW image and a reduced ADC value [25]. Compared with T1WI-DCE, SS-EPI DWI shows great accuracy, sensitivity and specificity in diagnosing myometrial invasion of endometrial carcinoma [26]. The fusion of SS-EPI DWI and T2WI images are also helpful for anatomical location and improve the accuracy of myometrial invasion of endometrial carcinoma. However, the distortion and blurring that are proportional to the 
Table 4 Diagnostic test evaluation indexes for judging myometrial invasion by RESOLVE DWI and SS-EPI DWI.

\begin{tabular}{|c|c|c|c|c|c|c|}
\hline \multirow[t]{2}{*}{ Parameters } & \multicolumn{2}{|c|}{ Intramucosal lesion } & \multicolumn{2}{|c|}{$<50 \%$ superficial invasion } & \multicolumn{2}{|c|}{$\geq \mathbf{5 0} \%$ myometrial invasion } \\
\hline & Resolve DWI & SS-EPI DWI & Resolve DWI & SS-EPI DWI & Resolve DWI & SS-EPI DWI \\
\hline $\begin{array}{l}\text { Sensitivity } \\
\text { ( } 95 \% \text { confidence interval) }\end{array}$ & $\begin{array}{l}4 / 6(66.67 \%) \\
(0.289,1.044)\end{array}$ & $\begin{array}{l}4 / 6(66.67 \%) \\
(0.289,1.044)\end{array}$ & $\begin{array}{l}14 / 18(77.78 \%) \\
(0.586,0.970)\end{array}$ & $\begin{array}{l}6 / 18(33.33 \%) \\
(0.116,0.551)\end{array}$ & $\begin{array}{l}4 / 6(66.67 \%) \\
(0.289,1.044)\end{array}$ & $\begin{array}{l}1 / 6(16.67 \%) \\
(-0.132,0.465)\end{array}$ \\
\hline Specificity & $22 / 24(91.67 \%)$ & 16/24 (66.67\%) & $8 / 12(66.67 \%)$ & $5 / 12(41.67 \%)$ & $22 / 24(91.67 \%)$ & 20/24 (83.33\%) \\
\hline Accuracy & $26 / 30(86.67 \%$ & $20 / 30(66.67 \%)$ & $22 / 30(73.33 \%)$ & $11 / 30(36.67 \%)$ & $26 / 30(86.67 \%)$ & $21 / 30(70 \%)$ \\
\hline $\begin{array}{l}\text { Positive predictive value } \\
\text { (95\% confidence interval) }\end{array}$ & $\begin{array}{l}4 / 6(66.67 \%) \\
(0.289,1.044)\end{array}$ & $\begin{array}{l}4 / 12(33.33 \%) \\
(0.067,0.600)\end{array}$ & $\begin{array}{l}14 / 18(77.78 \%) \\
(0.617,0.938)\end{array}$ & $\begin{array}{l}6 / 13(46.15 \%) \\
(0.191,0.733)\end{array}$ & $\begin{array}{l}4 / 6(66.67 \%) \\
(0.289,1.044)\end{array}$ & $\begin{array}{l}1 / 5(20 \%) \\
(-0.151,0.551)\end{array}$ \\
\hline Negative predictive value & $22 / 24(91.67 \%)$ & 16/18 (88.89\%) & $8 / 12(66.67 \%)$ & $5 / 17$ (29.41\%) & 22/24 (91.67\%) & $20 / 25(80 \%)$ \\
\hline Positive likelihood ratio & 8.004 & 2.000 & 2.333 & 0.571 & 8 & 1 \\
\hline Negative likelihood ratio & 0.364 & 0.500 & 0.333 & 1.600 & 0.364 & 1 \\
\hline
\end{tabular}

RESOLVE readout segmentation of long variable echo-trains, DWI diffusion-weighted imaging, SS-EPI single-shot echo-planar imaging

matrix size in SS-EPI DWI still limit the application of this technique for the acquisition of high-resolution images [27]. RESOLVE is a new RS-EPI technique and integrates this method with two-dimensional navigator correction to correct phase errors. The MR image quality is a key point of detecting and evaluating a lesion. So we evaluated the two kinds of DWI images in terms of geometric distortion, image blurring, artifacts, lesion display, and overall image quality. Our study demonstrated that RESOLVE DWI can obtain high-resolution images with less geometric distortion and artifacts. Another study of pelvic diseases had similar results. Thirty patients (containing three for cervical/endometrial cancer) underwent pelvic MRI with both SS-EPI and RS-EPI DWI. The study confirmed that RS-EPI DWI images showed better image quality compared to the SS-EPI technique at $3 \mathrm{~T}[20]$.

Quantitative evaluation of DWI image quality includes SNR, CNR and ADC. The SNR is an important quantity used to describe the performance of an MRI system. The most commonly used technique is to determine the signal intensity in the tissue of interest and to measure the noise intensity in the image background. Gassert FT

Table 5 The staging of 30 endometrial cancer patients by RESOLVE and SS-EPI DWI technology

\begin{tabular}{llll}
\hline FIGO & N & \multicolumn{2}{l}{ The number of accurate staging } \\
\cline { 3 - 4 } & & Resolve DWI & SS-EPI DW \\
\hline IA & 21 & 20 & 18 \\
IB & 4 & 3 & 1 \\
II & 1 & 1 & 0 \\
IIIA & 1 & 0 & 0 \\
IIIB & 2 & 1 & 0 \\
IIIC & 0 & 0 & 0 \\
IV & 1 & 1 & 1 \\
\hline
\end{tabular}

RESOLVE readout segmentation of long variable echo-trains, SS-EPI single-shot echo-planar imaging, DWI diffusion-weighted imaging, FIGO International Federation of Gynecology and Obstetrics et al. [28] and Gourtsoyianni S et al. [29] in their research applied this method, which is consistent with ours. The DWI images produced by the RESOLVE sequence showed higher SNR and CNR values compared with SS-EPI in endometrial carcinoma. This suggested that SI difference among images of different tissues was more obvious in RESOLVE DWI images. This feature may be convenient for identifying lesions. ADC value is related to the acquisition technique, including magnetic field strength, gradient performance, respiratory compensation technology, and the choice of b-values [3033]. The ADC values of endometrial cancer lesions had no statistical difference between RESOLVE and SS-EPI DWI images in our study. This indicated that RESOLVE DWI technology had no effect on the ADC values. In fact, RESOLVE DWI had advantages in measuring ADC value in other lesions. Higher specificity and better reproducibility of ADC measurements were found for coronal RESOLVE DWI in acute optic neuritis patients [34]. RESOLVE offered more accurate ADC values of sinonasal lesions than SS-EPI [13].

The results of the quantitative and qualitative analysis indicate that the image quality of RESOLVE DWI is better than that of SS-EPI DWI. The technique of RESOLVE DWI has proven to provide better detection and image quality in rectal, prostate, kidney, neck, and breast compared with SS-EPI DWI [15, 16, 18, 35, 36]. Our study also supports this conclusion.

The RESOLVE DWI typically is used to get higher resolution images. Our study found RESOLVE DWI images were more helpful to judge myometrium invasion in endometrial cancer. Although only the data in $95 \%$ confidence interval of sensitivity show that RESOLVE DWI is valuable in judging $<50 \%$ superficial invasion. The values of specificity, PPV, NPV, and positive likelihood ratio in the RESOLVE DWI group were all higher than those in the SS-EPI DWI group. This may be related to our small sample study. The depth of myometrial invasion represents a prognostic factors in 


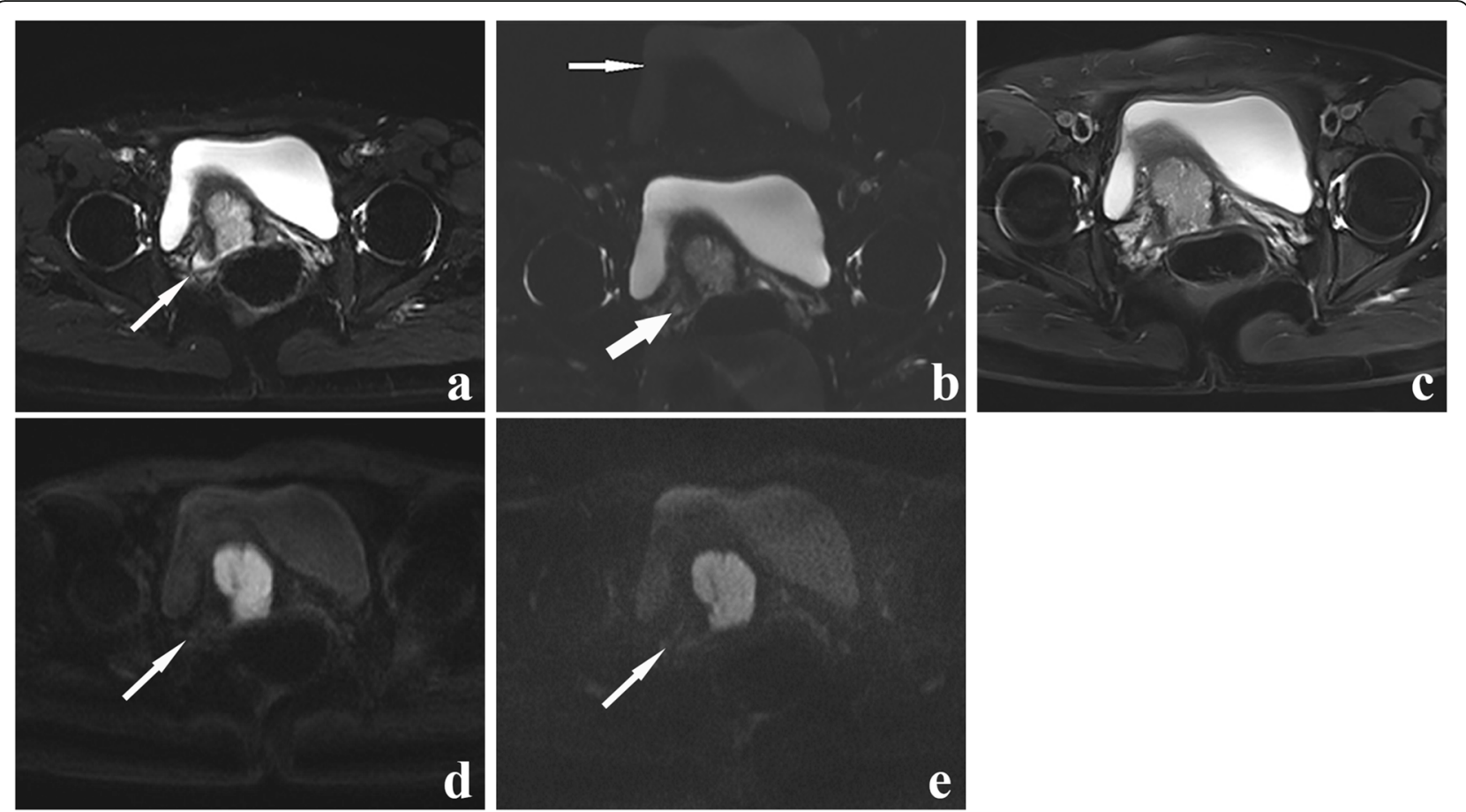

Fig. 5 A 54-year-old woman with endometrial cancer (endometrioid carcinoma, moderately differentiated, superficial myometrial invasion). a An axial RESOLVE DWI image $\left(b=0 \mathrm{~s} / \mathrm{mm}^{2}\right)$. b an axial SS-EPI DWI image $\left(b=0 \mathrm{~s} / \mathrm{mm}^{2}\right)$. $\mathbf{c}$ an axial T2WI image. $\mathbf{d}$ An axial RESOLVE DWI image $(b=$ $\left.1000 \mathrm{~s} / \mathrm{mm}^{2}\right)$. e an axial SS-EPI DWI image $\left(b=1000 \mathrm{~s} / \mathrm{mm}^{2}\right)$. The right side of the uterus is presented as a hyperintense signal on (a) (white arrow). (b) showing only a slightly higher signal intensity in the lesion (thick arrow). This suggested endometrial cancer on the right side of the uterus. The artifacts were seen on (b) (thin white arrow). As indicated by the white arrow, the lesion can be seen more clearly in (d) than in (e). Therefore, this case of stage IIIB endometrioid adenocarcinoma was accurately diagnosed by RESOLVE DWI

endometrial cancer, correlating with lymph node metastases and overall patient survival [37, 38]. The lymph node metastasis rate of the FIGO IA stage and IB stage was 3 and $46 \%$ respectively [39]. Tumor invasion to greater than $50 \%$ of the myometrial thickness translates to a six to seven times greater risk of pelvic and paraaortic lymph node metastases [40]. Therefore, accurate preoperative assessment of myometrial invasion by RESOLVE DWI is crucial to the choice of surgical approach and prognostic evaluation in endometrial cancer.

Errors in the assessment of myometrial invasion can occur in larger polypoid tumors, leiomyomas, congenital anomalies and very small atrophic uterus [41]. Compared with SS-EPI, RESOLVE DWI provided significantly better imaging quality and comparable diagnostic performance in detection of the depth of myometrial invasion. These features may be helpful for preoperative tumor staging. A study on preoperative staging of $68 \mathrm{pa-}$ tients with rectal cancer showed that the accuracy, sensitivity and specificity of RESOLVE DWI were higher than those of SS-EPI DWI [42]. RESOLVE DWI has clinical significance value in preoperative staging of rectal cancer and appropriate treatment options. Although we did not find the statistical differences between the two DWI technologies in clinical staging, RESOLVE DWI showed the potential benefits of clinical staging. A stage IIIB endometrial adenocarcinoma patient was correctly staged with RESOLVE DWI, while was underestimated with SS-EPI DWI in our study.

There were several limitations in the study. First, the image quality of RESOLVE DWI and SS-EPI DWI was evaluated only in the axial plane. The combination of the sagittal and coronal images will make the results more accurate. Secondly, we found that the MRI imaging of endometrial carcinoma lesions could not be completely avoided some affecting factors. These factors include the size of endometrial cancer lesions, secretion of endometrial mucus, and the peristalsis of the intestinal canal. We need to expand the sample size, spend more time studying sequence conditions, and prepare patients for the intestinal tract to reduce the impact of these factors. Finally, the echo times of the two DWI sequences are significantly different (reflected in Table 1), because the $3.0 \mathrm{~T}$ Siemens MRI scanner limits the settings. Thus, this difference prevents us from fairly comparing the SNR values of the two sequences. In addition, estimating the noise from the background requires a spatially homogeneous distribution of noise over the whole image. Although we adopt the method of measuring 3 times and taking the average, it may be difficult to 
avoid the error caused by the uneven lesion. Regardless of the above-mentioned limitations, we believe that the RESOLVE DWI has further advantages over the SS-EPI DWI in the evaluation of endometrial carcinoma.

\section{Conclusion}

RESOLVE DWI can provide high quality images of endometrial carcinoma, which is helpful for the accurate assessment of myometrial invasion in endometrial carcinoma. The precise estimate will be helpful to select the suitable endometrioid adenocarcinoma patients for conservative treatment and monitoring the effects. It is also helpful to select the appropriate operative type and reduce the occurrence of surgical complications for the endometrial cancer patients [43].

\section{Supplementary information}

Supplementary information accompanies this paper at https://doi.org/10 1186/s40644-020-00346-7.

Additional file 1: Supplementary Table 1. FIGO Staging with

Corresponding MR imaging.

Additional file 2: Supplementary Table 2. MRI criteria for the assessment of myometrial invasion.

Additional file 3: Supplementary Table 3. The operation methods in 30 patients.

Additional file 4: Supplementary Table 4. Correlation analysis of qualitative scores between two observers.

Additional file 5: Supplementary Table 5. Comparison of SS-EPI DWI and RESOLVE DWI in diagnosis of myometrial invasion and pathological results of endometrial carcinoma.

\section{Abbreviations}

ADC: Apparent diffusion coefficient; CNR: Contrast to noise ratio; DWMRI: Diffusion-weighted MRI; DCE-MRI: Dynamic contrast-enhanced MRl; DWI: Diffusion-weighted imaging; FIGO: International Federation of Obstetrics and Gynecology; Gd-DTPA: Gadopentetate Dimeglumine; MRI: Magnetic resonance imaging; NPV: Negative predictive value; PPV: Positive predictive value; RS-EPI: Readout-segmented echo-planar imaging; RESOLVE DWI: Readout segmentation of long variable echo-trains diffusion-weight imaging; ROI: Region of interest; SS-EPI: Single-shot echoplanar imaging; SNR: Signal to noise ratio; T1WI: T1-weighted imaging; T2WI: T2-weighted imaging; TSE: Turbo spin echo

\section{Acknowledgments}

The authors thank Jing Deng for her assistance in manuscript preparation.

\section{Authors' contributions}

MNX and ZR: manuscript preparation, literature research, and data analysis. DJB: imaging data collection and analysis. DL: imaging data collection. LY, FZ and RH: data acquisition. ZBZ: literature research. SS: manuscript editing. CF: study conception and design, manuscript review and guarantor of integrity of the entire study. All authors have read and approved the final manuscript.

\section{Funding}

This work was supported by the National Natural Science Foundation Project of China (81771546), Hunan Provincial Department of Finance and Education Project (2018) No. 33: (2050205-30299-50502) and Hunan Science and Technology Innovation Project (2018SK52508).

\section{Availability of data and materials}

The datasets used and/or analyzed during the current study are available from the corresponding author on reasonable request.

\section{Ethics approval and consent to participate}

The study protocol was approved by the institutional review board and written informed consent was obtained from all participants.

\section{Consent for publication}

Not applicable.

\section{Competing interests}

The authors declare that they have no competing interests.

\section{Author details}

'Department of Obstetrics and Gynecology, The Second Xiangya Hospital, Central South University, Changsha, No.139 Renmin Road, Changsha, Hunan 410011, PR China. ${ }^{2}$ Department of Radiology, The Second Xiangya Hospital, Central South University, Changsha, Hunan, PR China.

Received: 19 February 2020 Accepted: 15 September 2020

Published online: 21 September 2020

\section{References}

1. Yue W, Meng N, Wang J, Liu W, Wang X, Yan M, Han D, Cheng J. Comparative analysis of the value of diffusion kurtosis imaging and diffusion-weighted imaging in evaluating the histological features of endometrial cancer. Cancer Imaging. 2019;19(1):9.

2. Zhang Q, Yu X, Lin M, Xie L, Zhang M, Ouyang H, Zhao X. Multi-b-value diffusion weighted imaging for preoperative evaluation of risk stratification in early-stage endometrial cancer. Eur J Radiol. 2019;119:108637.

3. Rauch GM, Kaur H, Choi H, Ernst RD, Klopp AH, Boonsirikamchai P, Westin SN, Marcal LP. Optimization of MR imaging for pretreatment evaluation of patients with endometrial and cervical cancer. Radiographics. 2014;34(4): 1082-98.

4. Beddy P, Moyle P, Kataoka M, Yamamoto AK, Joubert I, Lomas D, Crawford $R$, Sala E. Evaluation of depth of myometrial invasion and overall staging in endometrial cancer: comparison of diffusion-weighted and dynamic contrast-enhanced MR imaging. Radiology. 2012;262(2):530-7.

5. Kawaguchi M, Kato H, Hatano Y, Mizuno N, Furui T, Morishige K, Hara A, Goshima S, Matsuo M. Inchworm sign of endometrial cancer on diffusionweighted MRI: radiology-pathology correlation. Clin Radiol. 2018;73(10):907. e909-14.

6. Guo Y, Wang P, Wang P, Gao W, Li F, Yang X, Ni H, Shen W, Guo Z. Myometrial invasion and overall staging of endometrial carcinoma: assessment using fusion of T2-weighted magnetic resonance imaging and diffusion-weighted magnetic resonance imaging. Onco Targets Ther. 2017; 10:5937-43.

7. Lin G, Ng K-K, Chang C-J, Wang J-J, Ho K-C, Yen T-C, Wu T-I, Wang C-C, Chen $Y-R$, Huang $Y-T$, et al. Myometrial invasion in endometrial cancer: diagnostic accuracy of diffusion-weighted 3.0-T MR imaging--initial experience. Radiology. 2009:250(3):784-92.

8. Das SK, Niu XK, Wang JL, Zeng LC, Wang WX, Bhetuwal A, Yang HF. Usefulness of DWI in preoperative assessment of deep myometrial invasion in patients with endometrial carcinoma: a systematic review and metaanalysis. Cancer Imaging. 2014;14(1):32.

9. Gil RT, Cunha TM, Horta M, Alves I. The added value of diffusion-weighted imaging in the preoperative assessment of endometrial cancer. Radiol Bras. 2019:52(4):229-36.

10. Sala E, Rockall A, Rangarajan D, Kubik-Huch RA. The role of dynamic contrast-enhanced and diffusion weighted magnetic resonance imaging in the female pelvis. Eur J Radiol. 2010;76(3):367-85.

11. Lu P, Tian G, Liu X, Wang F, Zhang Z, Sha Y. Differentiating Neuromyelitis Optica-related and multiple sclerosis-related acute optic neuritis using conventional magnetic resonance imaging combined with readoutsegmented Echo-planar diffusion-weighted imaging. J Comput Assist Tomogr. 2018;42(4):502-9.

12. Wisner DJ, Rogers N, Deshpande VS, Newitt DN, Laub GA, Porter DA, Kornak $J$, Joe BN, Hylton NM. High-resolution diffusion-weighted imaging for the separation of benign from malignant BI-RADS $4 / 5$ lesions found on breast MRI at 3T. J Magn Reson Imaging. 2014;40(3):674-81.

13. Zhao M, Liu Z, Sha Y, Wang S, Ye X, Pan Y, Wang S. Readout-segmented echo-planar imaging in the evaluation of sinonasal lesions: a comprehensive comparison of image quality in single-shot echo-planar imaging. Magn Reson Imaging. 2016;34(2):166-72. 
14. Kim TH, Baek MY, Park JE, Ryu YJ, Cheon JE, Kim IO, Choi YH. Comparison of DWI methods in the pediatric brain: PROPELLER Turbo spin-Echo imaging versus readout-segmented Echo-planar imaging versus single-shot Echoplanar imaging. AJR Am J Roentgenol. 2018;210(6):1352-8.

15. Friedli I, Crowe LA, de Perrot T, Berchtold L, Martin PY, de Seigneux S, Vallee JP. Comparison of readout-segmented and conventional single-shot for echo-planar diffusion-weighted imaging in the assessment of kidney interstitial fibrosis. J Magn Reson Imaging. 2017:46(6):1631-40.

16. Fan WX, Chen XF, Cheng FY, Cheng YB, Xu T, Zhu WB, Zhu XL, Li GJ, Li S. Retrospective analysis of the utility of multiparametric MRI for differentiating between benign and malignant breast lesions in women in China. Medicine. 2018;97(4):e9666

17. Pham TT, Liney G, Wong K, Rai R, Lee M, Moses D, Henderson C, Lin M, Shin JS, Barton MB. Study protocol: multi-parametric magnetic resonance imaging for therapeutic response prediction in rectal cancer. BMC Cancer. 2017;17(1):465.

18. Liney GP, Holloway L, Al Harthi TM, Sidhom M, Moses D, Juresic E, Rai R, Manton DJ. Quantitative evaluation of diffusion-weighted imaging techniques for the purposes of radiotherapy planning in the prostate. $\mathrm{Br}$ J Radiol. 2015;88(1049):20150034

19. Li H, Liu L, Shi Q, Stemmer A, Zeng H, Li Y, Zhang M. Bladder cancer: detection and image quality compared among iShim, RESOLVE, and ss-EPI diffusion-weighted MR imaging with high b value at 3.0 T MRI. Medicine. 2017;96(50):e9292.

20. Thian YL, Xie W, Porter DA, Weileng Ang B. Readout-segmented echoplanar imaging for diffusion-weighted imaging in the pelvis at 3T-A feasibility study. Acad Radiol. 2014;21(4):531-7.

21. Alhassan AA, Alqadhib EM, Taha NW, Alahmari RA, Salam M, Almutairi AF. The relationship between addiction to smartphone usage and depression among adults: a cross sectional study. BMC Psychiatry. 2018;18(1):148.

22. Dietrich O, Raya JG, Reeder SB, Reiser MF, Schoenberg SO. Measurement of signal-to-noise ratios in MR images: influence of multichannel coils, parallel imaging, and reconstruction filters. J Magn Reson Imaging. 2007;26(2):37585.

23. Kishida Y, Koyama H, Seki S, Yoshikawa T, Kyotani K, Okuaki T, Sugimura K, Ohno Y. Comparison of fat suppression capability for chest MR imaging with Dixon, SPAIR and STIR techniques at 3 tesla MR system. Magn Reson Imaging. 2018;47:89-96.

24. Nougaret S, Lakhman Y, Vargas HA, Colombo PE, Fujii S, Reinhold C, Sala E. From staging to prognostication: achievements and challenges of MR imaging in the assessment of endometrial Cancer. Magn Reson Imaging Clin N Am. 2017;25(3):611-33.

25. Bakir B, Sanli S, Bakir VL, Ayas S, Yildiz SO, lyibozkurt AC, Kartal MG, Yavuz E. Role of diffusion weighted MRI in the differential diagnosis of endometrial cancer, polyp, hyperplasia, and physiological thickening. Clin Imaging. 2017; 41:86-94.

26. Rechichi G, Galimberti S, Signorelli M, Perego P, Valsecchi MG, Sironi S. Myometrial invasion in endometrial cancer: diagnostic performance of diffusion-weighted MR imaging at 1.5-T. Eur Radiol. 2010;20(3):754-62.

27. Le Bihan D, Poupon C, Amadon A, Lethimonnier F. Artifacts and pitfalls in diffusion MRI. J Magn Reson Imaging. 2006;24(3):478-88.

28. Gassert FT, Gassert FG, Topping GJ, Rummeny EJ, Wildgruber M, Meier R, Kimm MA. SNR analysis of contrast-enhanced MR imaging for early detection of rheumatoid arthritis. PLoS One. 2019;14(3):e0213082.

29. Gourtsoyianni S, Papanikolaou N, Yarmenitis S, Maris T, Karantanas A, Gourtsoyiannis N. Respiratory gated diffusion-weighted imaging of the liver: value of apparent diffusion coefficient measurements in the differentiation between most commonly encountered benign and malignant focal liver lesions. Eur Radiol. 2008;18(3):486-92.

30. Rosenkrantz AB, Oei M, Babb JS, Niver BE, Taouli B. Diffusion-weighted imaging of the abdomen at 3.0 tesla: image quality and apparent diffusion coefficient reproducibility compared with 1.5 tesla. J Magn Reson Imaging. 2011;33(1):128-35.

31. Sasaki M, Yamada K, Watanabe Y, Matsui M, Ida M, Fujiwara S, Shibata E. Acute stroke imaging standardization group-Japan I: variability in absolute apparent diffusion coefficient values across different platforms may be substantial: a multivendor, multi-institutional comparison study. Radiology. 2008;249(2):624-30.

32. Girometti R, Furlan A, Esposito G, Bazzocchi M, Como G, Soldano F, Isola M, Toniutto P, Zuiani C. Relevance of b-values in evaluating liver fibrosis: a study in healthy and cirrhotic subjects using two single-shot spin-echo echo-planar diffusion-weighted sequences. J Magn Reson Imaging. 2008; 28(2):411-9.

33. Kwee TC, Takahara T, Koh D-M, Nievelstein RAJ, Luijten PR. Comparison and reproducibility of $A D C$ measurements in breathhold, respiratory triggered, and free-breathing diffusion-weighted MR imaging of the liver. J Magn Reson Imaging. 2008;28(5):1141-8.

34. Lu P, Sha Y, Wan H, Wang F, Tian G. Role of coronal high-resolution diffusion-weighted imaging in acute optic neuritis: a comparison with axial orientation. Neuroradiology. 2017;59(8):737-45.

35. Tang C, Lin M-B, Xu J-L, Zhang L-H, Zuo X-M, Zhang Z-S, Liu M-X, Xu J-M. Are ADC values of readout-segmented echo-planar diffusion-weighted imaging (RESOLVE) correlated with pathological prognostic factors in rectal adenocarcinoma? World J Surg Oncol. 2018;16(1):138.

36. Song C, Cheng P, Cheng J, Zhang Y, Sun M, Xie S, Zhang X. Differential diagnosis of nasopharyngeal carcinoma and nasopharyngeal lymphoma based on DCE-MRI and RESOLVE-DWI. Eur Radiol. 2020;30(1):110-8.

37. Ben-Shachar I, Vitellas KM, Cohn DE. The role of MRI in the conservative management of endometrial cancer. Gynecol Oncol. 2004;93(1):233-7.

38. Kinkel K, Forstner R, Danza FM, Oleaga L, Cunha TM, Bergman A, Barentsz JO, Balleyguier C, Brkljacic B, Spencer JA, et al. Staging of endometrial cancer with MRI: guidelines of the European Society of Urogenital Imaging. Eur Radiol. 2009;19(7):1565-74.

39. Boronow RC, Morrow CP, Creasman WT, Disaia PJ, Silverberg SG, Miller A, Blessing JA. Surgical staging in endometrial cancer: clinical-pathologic findings of a prospective study. Obstet Gynecol. 1984;63(6):825-32.

40. Larson DM, Connor GP, Broste SK, Krawisz BR, Johnson KK. Prognostic significance of gross myometrial invasion with endometrial cancer. Obstet Gynecol. 1996;88(3):394-8.

41. Sala E, Wakely S, Senior E, Lomas D. MRI of malignant neoplasms of the uterine corpus and cervix. AJR Am J Roentgenol. 2007;188(6):1577-87.

42. Wu JK. High resolution MRI and diffusion-weighted imaging in preoperation staging of primary rectal Cancer. 1st ed. Guangzhou: Southern Medical University; 2016. p. 71.

43. Sala E, Rockall A, Kubik-Huch RA. Advances in magnetic resonance imaging of endometrial cancer. Eur Radiol. 2011;21(3):468-73.

\section{Publisher's Note}

Springer Nature remains neutral with regard to jurisdictional claims in published maps and institutional affiliations.
Ready to submit your research? Choose BMC and benefit from:

- fast, convenient online submission

- thorough peer review by experienced researchers in your field

- rapid publication on acceptance

- support for research data, including large and complex data types

- gold Open Access which fosters wider collaboration and increased citations

- maximum visibility for your research: over $100 \mathrm{M}$ website views per year

At BMC, research is always in progress.

Learn more biomedcentral.com/submissions 